

\title{
Characterization of the evaporation of interacting droplets using combined optical techniques
}

\author{
Lionel Perrin, Guillaume Castanet, Fabrice Lemoine
}

\section{To cite this version:}

Lionel Perrin, Guillaume Castanet, Fabrice Lemoine. Characterization of the evaporation of interacting droplets using combined optical techniques. Experiments in Fluids, 2015, 56 (2), pp.29. 10.1007/s00348-015-1900-3 . hal-01417340

\section{HAL Id: hal-01417340 \\ https://hal.univ-lorraine.fr/hal-01417340}

Submitted on 30 Jul 2017

HAL is a multi-disciplinary open access archive for the deposit and dissemination of scientific research documents, whether they are published or not. The documents may come from teaching and research institutions in France or abroad, or from public or private research centers.
L'archive ouverte pluridisciplinaire HAL, est destinée au dépôt et à la diffusion de documents scientifiques de niveau recherche, publiés ou non, émanant des établissements d'enseignement et de recherche français ou étrangers, des laboratoires publics ou privés. 


\title{
Characterization of the evaporation of interacting droplets using combined optical techniques
}

\author{
Lionel Perrin • Guillaume Castanet · Fabrice Lemoine
}

\begin{abstract}
A monodisperse droplet stream is injected into a high-temperature enclosure supplied with air heated up to $540{ }^{\circ} \mathrm{C}$. The two-color laser-induced fluorescence (2cLIF) is used for measuring the droplet temperature. The liquid fuel is seeded by pyrromethene 597-C8, which is a temperature-sensitive fluorescent dye. Calibration tests are performed for different types of fuels including ethanol and several alkanes and some of their mixtures. Morphologydependent resonances (MDRs) are identified as a possible adverse effect for temperature measurements. Due to MDRs, lasing of pyrromethene 597-C8 may occur within fluorescent droplets and affect drastically the fluorescence signal upon which temperature measurement relies. The determination of the droplet size and velocity is achieved by means of quantitative shadow imaging. A double cavity PIV laser is focused on a piece of PMMA doped with a fluorescent dye to produce the background illumination of the droplets. A PIV camera is used to capture the drop motion between the pulses of the laser cavities. A large range of initial distance parameters (the ratio between the inter-droplet distance and the droplet diameter) is explored for different liquid fuels (ethanol, isohexane, $n$-heptane, $n$-decane, $n$-dodecane) and their mixtures. To put forward the effects of the interactions between the droplets, size and temperature measurements are compared to the isolated droplet whose evolution can be predicted with the use of classical models. Comparisons reveal that the inter-droplet
\end{abstract}

L. Perrin · G. Castanet $(\triangle) \cdot$ F. Lemoine

Université de Lorraine, LEMTA, UMR 7563,

F-54500 Vandoeuvre-Lès-Nancy, France

e-mail: guillaume.castanet@univ-lorraine.fr

L. Perrin · G. Castanet · F. Lemoine

CNRS, LEMTA, UMR 7563, F-54500 Vandoeuvre-Lès-Nancy,

France spacing and also the fuel volatility play an important role in the reduction of the heat and mass transfers for these interacting droplets. Finally, the ability of the 2cLIF techniques to address the case of multicomponent droplet is also demonstrated.

\section{Introduction}

Liquid fuels are often used in combustion systems for propulsion of aircrafts, rockets or automobiles. In the frame of the reduction in pollutant emissions, research is now oriented on optimizing the injection system used in the combustion chambers. One of the dominant factors is the droplet concentration produced near the outlet of the injector, since the zone of high fuel concentration, despite being necessary for the ignition, induces high levels of pollutant emission, incomplete oxidation and unburned fuel. The present paper deals with a fundamental study of droplet evaporation in an idealized configuration where interactions occur between closely spaced droplets. A line of equally spaced and monosized drops is injected into a high-temperature enclosure. Important parameters such as the distance between the droplets are modified in order to characterize their influence on the droplet evaporation. Monodisperse droplet streams have been used in previous studies of droplet evaporation (Castanet et al. 2005, 2011; Deprédurand et al. 2010; Virepinte et al. 2000). These studies emphasized the role of the dimensionless spacing parameter $C$, defined as the ratio between the droplet spacing $L$ and the droplet size $d$. For low values of $C$, the heating and evaporation rates are reduced in comparison with the isolated droplet whose evolution can be predicted using classical models (Abramzon and Sirignano 1989). The mechanism of these interactions between the droplets is still not clear. 
For rapidly vaporizing droplets, it can be assumed that the vapor released by the drops is transported in the vicinity of neighbor droplets reducing the gradients of vapor concentration near their surface and thus their vaporization rate. Castanet et al. (2005) studied the evaporation of ethanol droplet streams in a combusting flow. They observed significant reductions in the heat and mass transfer rates for $C$ lower than 9. More recently, Deprédurand et al. (2010) extended these works to several liquid fuels by conducting experiments in air heated up to $350{ }^{\circ} \mathrm{C}$. They demonstrated that the reduction in the heat and mass transfers can differ between the liquid fuels even at identical distance parameters and Reynolds numbers. They also observed significantly greater reductions than those reported by Castanet et al. (2005) for combusting droplets. These differences were attributed to the fuel volatility. For volatile fuels, they assumed that the Stefan flow induced by the ejection of vapor at the drop surface can be strong enough to have an incidence on the vapor dispersion in the outer flow.

Practical fuels are also composed of hundreds of chemical species. From an experimental point of view, a challenge is to measure thermochemical quantities such as temperature and composition of multicomponent droplets. Different optical methods have been developed in the past to measure temperature. Without being exhaustive, one can mention Raman spectroscopy, morphology-dependent resonances (MDRs), rainbow refractometry and laser-induced fluorescence (LIF; Lemoine and Castanet 2013). Each method has its own advantages and limitations depending on its basic principles. For instance, the fluorescence signal emitted by temperature-sensitive fluorophores generally exhibits some dependence on the composition in addition to the temperature. In fact, the chemical environment alters some spectroscopic properties of the dye (generally very diluted). In particular, it can modify collisional quenching energy distribution in the excited state. The feasibility of temperature measurements based on LIF is presently more thoroughly investigated for several mixtures made of alkane species (isohexane, $n$-heptane, $n$-decane, $n$-dodecane and $n$-hexadecane). For characterizing the droplet evaporation and velocity, temperature measurements are combined with an efficient method based on quantitative shadow imaging.

\section{Experimental setup and optical techniques}

A monodisperse droplet stream is generated by the disintegration of a liquid jet undergoing vibrations from a piezoceramic. The droplets are injected into an insulated enclosure supplied with hot air passing through an electrical heater (Fig. 1). In order to limit the thermal losses, a resistive electrical wire is inserted within the wall of the

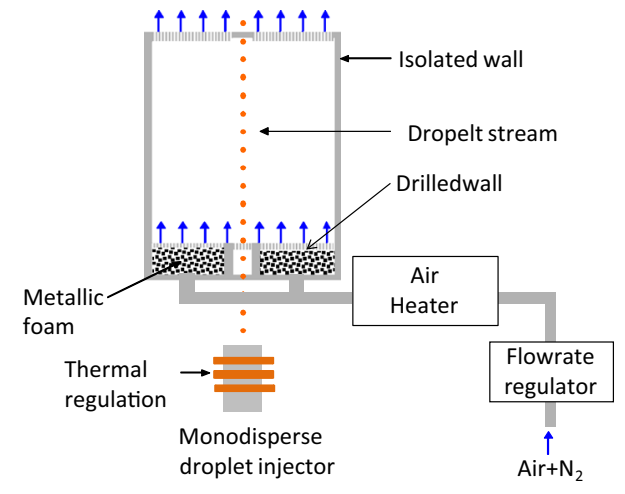

Fig. 1 Schema of the experimental setup: heated enclosure and droplet monodisperse stream

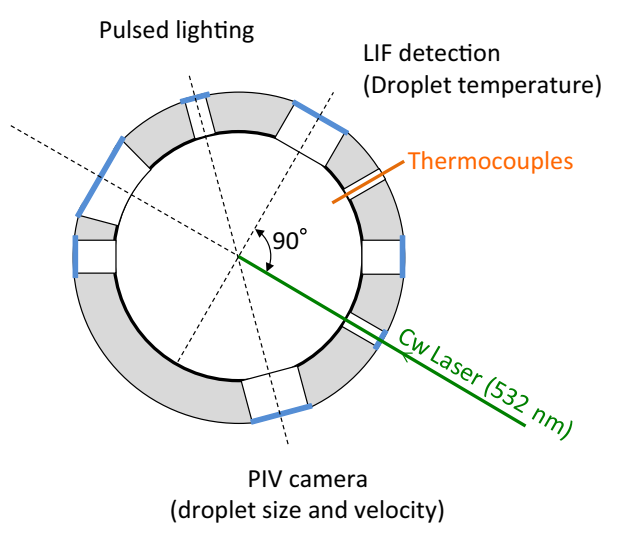

Fig. 2 Optical access for the implementation of the different optical techniques

enclosure so that the wall can be regulated at the same temperature as the incoming air (Fig. 1). Temperatures up to $540{ }^{\circ} \mathrm{C}$ can be reached inside the chamber. Nitrogen is added to the incoming air to reduce the fraction of oxygen and thus avoid any ignition of the fuel vapor. The air flow is quieted by forcing it to enter in the chamber through metallic foam and a drilled wall. The gas velocity is small (ranging between 0.1 and $0.3 \mathrm{~m} / \mathrm{s}$ ) compared to that of the droplets (typically a few $\mathrm{m} / \mathrm{s}$ ). The gas flow ensures an almost uniform temperature field in the chamber and also prevents vapor saturation. The high periodicity achieved with the monodisperse droplet stream allows converting the distance covered by the droplets after their injection into time, provided that the local velocity is measured. Three main parameters are measured at each investigated locations of the monodisperse droplet stream: the temperature, the velocity and the diameter of the droplets. To that end, quartz windows have been managed in the wall to offer several optical accesses (Fig. 2). 


\subsection{Droplet temperature measurements}

The two-color laser-induced fluorescence (2cLIF), initiated by Lavieille et al. (2001), is used to characterize the droplet temperature. The liquid fuel is seeded by a fluorescent dye, pyrromethene 597-C8, which has a fluorescence spectrum that is resonant with the wavelength of a frequencydoubled Nd-Yag laser at $532 \mathrm{~nm}$. Pyrromethene 597-C8 is easily soluble in alkanes contrary to other fluorescent dopants such as rhodamine B and Kiton red that are usually used for temperature measurement in aqueous solutions. Figure 3 shows the broadband emission spectrum of pyrromethene 597-C 8 dissolved into $n$-dodecane at several temperatures ranging from 20 to $100{ }^{\circ} \mathrm{C}$. A pronounced decay of the fluorescence signal with the temperature can be clearly observed. A sharp high-pass filter with a cutoff at $542 \mathrm{~nm}$ (optical density of 6) is utilized to record the spectra presented in Fig. 3 in order to avoid any perturbation of the laser wavelength. Doing so, a secondary emission peak around $535 \mathrm{~nm}$ was partially hidden. The decrease in this second peak with the temperature is less important than that in the main peak centered around $600 \mathrm{~nm}$ (Deprédurand et al. 2008). According to Lemoine and Castanet (2013), the fluorescence intensity $I_{\mathrm{f}, i}$ detected over a specific spectral band noted $i$ can be expressed as:

$I_{\mathrm{f}, i}=K_{\mathrm{opt}, i} K_{\mathrm{spec}, i} I_{0} C V_{c} f_{i}(T)$

In this expression, $C$ denotes the concentration of the fluorescent dye. $I_{0}$ is the intensity of the laser beam. $K_{\mathrm{opt}, i}$ is an optical constant that accounts for the efficiency of the detection, which includes the solid angle between

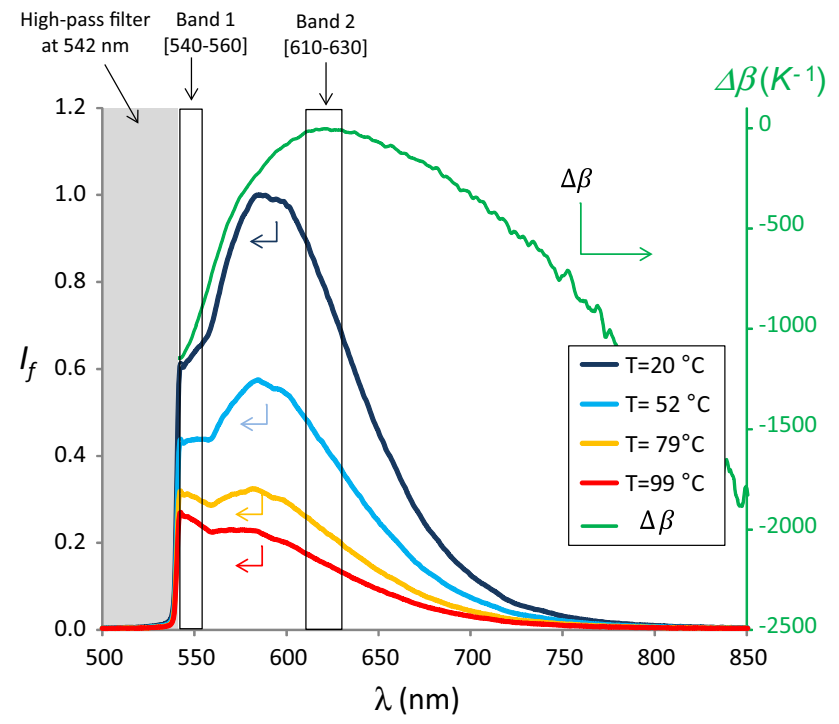

Fig. 3 Emission spectra of pyrromethene 597-C8 recorded at different temperatures. In green, the temperature sensitivity $\Delta \beta=-T^{2} \frac{\partial}{\partial T} \ln \left(I_{f}(\lambda) / I_{f}\left(\lambda_{0}\right)\right)$ with $\lambda_{0}=630 \mathrm{~nm}$ the detector and the emission source. $K_{\mathrm{spec}, i}$ is a constant depending on the spectroscopic properties of the fluorescent dye in its chemical environment. $K_{\mathrm{opt}, i}$ and $K_{\mathrm{spec}, i}$ are not affected by the temperature meaning that the dependence in temperature of the fluorescence signal is exclusively contained in $f_{i}(T)$. The measurement volume $V_{\mathrm{C}}$ is formed by the intersection between the laser beam, the detection solid angle and the droplet. The fluorescence signal varies with time when the drop crosses the laser beam and the detection volume. The shape and amplitude of the fluorescence signal from a moving droplet depend on many factors that are difficult to control such as the size of the droplet relative to the diameter of the laser beam and the droplet trajectory (if the droplet is off-centered relatively to the laser beam and the focal point of the detection optics). The most common methods for eliminating this problem are based on measurements of intensity ratios. The fluorescence ratio between fluorescence intensities collected on two separate spectral bands is given by:

$$
\begin{aligned}
& R_{12}(T)=\frac{I_{\mathrm{f}, 1}}{I_{\mathrm{f}, 2}}=\frac{K_{\mathrm{opt}, 1} K_{\mathrm{spec}, 1}}{K_{\mathrm{opt}, 2} K_{\mathrm{spec}, 2}} f_{12}(T) \text { with } \\
& f_{12}(T)=f_{1}(T) / f_{2}(T) .
\end{aligned}
$$

The use of a single reference point where the temperature is known allows eliminating the optical and spectroscopic constants in Eq. 2 meaning that $R_{12}(T) / R_{12}\left(T_{\text {ref }}\right)=$ $f_{12}(T) / f_{12}\left(T_{\text {ref }}\right)$. The ratio $R_{12}$ is independent on the dimensions of the intersection between the droplet, the laser excitation volume and the photon collection volume. The influence of the local laser excitation intensity and dye concentration are also totally eliminated. For accurately measuring the temperature, spectral bands of detection should be selected so that their sensibility to the temperature is highly different. The sensibility factor $\beta(\lambda)=-T^{2} \partial \ln I_{\mathrm{f}}(\lambda) / \partial T$ is plotted in Fig. 3. A marked difference in temperature sensitivity can be observed between the green-yellow region and the orange-red region of the emission spectrum. Temperature measurements can be therefore optimized by choosing the spectral bands that are indicated in Fig. 3. A schematic view of the 2 cLIF measurement system is presented in Fig. 4. The excitation volume of the fluorescence is formed by two crossing laser beams, which helps positioning the droplets at the beamweast of the laser beams to optimize the LIF signal intensity. A fiber-coupled achromatic doublet allows the fluorescence emission to be collected at right angle. Mie scattering by the droplets at the laser wavelength is eliminated using the already-mentioned sharp high-pass filter. A set of beam splitter and interference filters enables the fluorescence signal to be separated in the two spectral bands of interest. The fluorescence signal is then detected by means of two photomultiplier tubes equipped with rapid pre-amplifiers. 


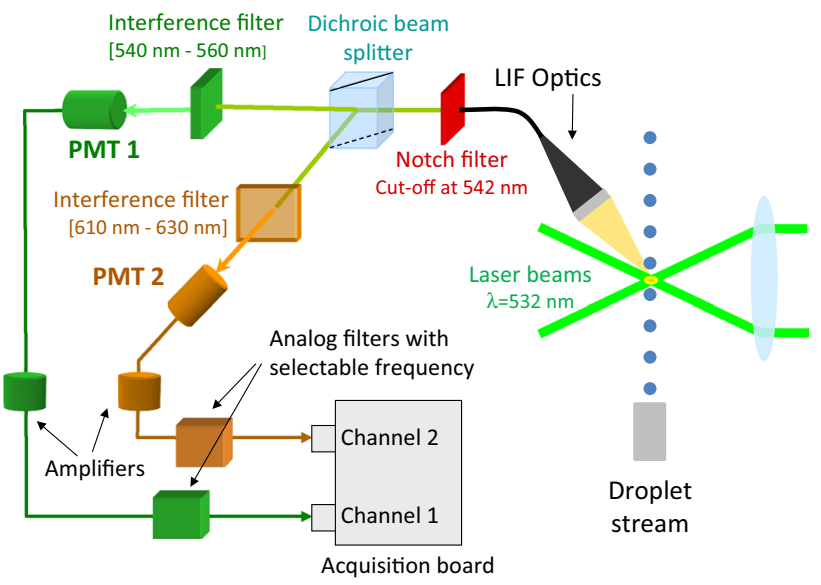

Fig. 4 Schematic view of the 2cLIF system for droplet temperature measurement

A calibration in temperature of the measurement system is performed in a liquid cell regulated in temperature. The liquid is progressively heated while measuring the fluorescence ratio $R_{12}$ and the liquid temperature by means of a thermocouple. To limit the reabsorption of the fluorescence by the dye, special care is taken to position the measurement volume at a short distance from the glass window of the calibration cell. Indeed, Eq. 1 does not account for the effects of the absorption of the laser beam and the reabsorption of fluorescence in the liquid medium. This approximation is acceptable for droplets given their very small size but may fail for measurements performed in a cell. Errors on the calibration may arise if the absorption coefficient of the dye is temperature dependent and if this temperature dependence is not the same for the two spectral bands of interest. Also, the wavelength-dependent absorption by the dye induces a filtering of the fluorescent light emerging in the direction of the detector. This spectral filtering can alter the profile of a detection band if the absorption coefficient significantly varies over the given band. It is accompanied by a modification of the overall temperature sensitivity of the fluorescence ratio. As reported by Deprédurand et al. (2008), the temperature dependence of the absorption coefficient is very weak in the case of pyrromethene 597-C8. The application of the Beer-Lambert law (taken $\varepsilon_{1}=2.3 \times 10^{3} \mathrm{~m}^{2} / \mathrm{mol}$ for the band [540-560 nm] and $\varepsilon_{2}=0.06 \times 10 \mathrm{~m}^{2} / \mathrm{mol}$ for the band [610-630 nm]) shows that the effect of reabsorption on the fluorescence ratio can be safely ignored if the probe volume is positioned at a distance of $1 \mathrm{~mm}$ or less from the windows of the cell for a dye concentration $c=5 \times 10^{-6} \mathrm{~mol} / \mathrm{L}$. Figure 5 presents the calibration curves obtained for different fuels including ethanol, isohexane, $n$-heptane, $n$-decane and $n$-dodecane, which have very different boiling temperature from $60{ }^{\circ} \mathrm{C}$ (isohexane) to $216{ }^{\circ} \mathrm{C}$ (n-dodecane).



Fig. 5 Calibration curves showing the effect of temperature on the signal ratio $R_{12}\left(T_{0}=25^{\circ} \mathrm{C}\right)$

It can be observed that the sensitivity to temperature of the fluorescence ratio is influenced by the liquid solvent even within the same group of chemical compounds such as alkanes. Temperature sensitivity $\left(\mathrm{d} R_{12} / \mathrm{d} T\right)$ is lower in $n$-heptane (about $0.7 \% /{ }^{\circ} \mathrm{C}$ at $25^{\circ} \mathrm{C}$ ) and higher in ethanol (about $1 \% /{ }^{\circ} \mathrm{C}$ at $25^{\circ} \mathrm{C}$ ). Interpretation for these differences is that intermolecular deactivations of the excited states (mainly by collisional quenching) are temperature-dependent processes that are by nature function of the quencher type and hence the liquid solvent containing the dye molecules. Based on previous works (Lavieille et al. 2004), an appropriate fitting of the calibration curves is provided by:

$f_{12}(T) \approx \exp \left(\frac{A}{T^{2}}+\frac{B}{T}+C\right)$,

where $A, B$ and $C$ are coefficients obtained experimentally from the calibration curves in Fig. 5. Estimated values of $A$ and $B$ are given in Table 1 for the different tested fuels. The adjusted value for $C$ in Eq. (3) is not significant, as it has to be determined along with $K_{\mathrm{opt}, 1} K_{\text {spec, } 1} / K_{\mathrm{opt}, 2} K_{\mathrm{spec}, 2}$ by doing a reference at a known temperature. The reference is usually taken very near the injection point so that the droplet temperature does not have time to change much between the injection and the reference. A thermocouple placed into the injector body allows measuring the liquid temperature at the injection outlet.

\subsection{Temperature measurements in mixtures}

Based on Fig. 5, some liquid fuels seem particularly well indicated to compose mixtures as pyrromethene 597-C8 
Table 1 Calibration coefficients obtained with the measurement system described in Fig. 5

\begin{tabular}{lll}
\hline & $A$ & $B$ \\
\hline 1 & 981,802 & $-7,263.1$ \\
2 & 362,233 & $-3,082.2$ \\
3 & 463,493 & $-3,574.3$ \\
4 & 759,718 & $-5,603.1$ \\
5 & 680,881 & $-5,113.1$ \\
\hline
\end{tabular}

1: Ethanol $\left(19^{\circ} \mathrm{C}<T<72^{\circ} \mathrm{C}\right)$

2: iso-Hexane $\left(15^{\circ} \mathrm{C}<T<50^{\circ} \mathrm{C}\right)$

3: $n$-Heptane $\left(15^{\circ} \mathrm{C}<T<89^{\circ} \mathrm{C}\right)$

4: $n$-Decane $\left(21^{\circ} \mathrm{C}<T<143{ }^{\circ} \mathrm{C}\right)$

5: $n$-Dodecane $\left(19^{\circ} \mathrm{C}<T<148^{\circ} \mathrm{C}\right)$

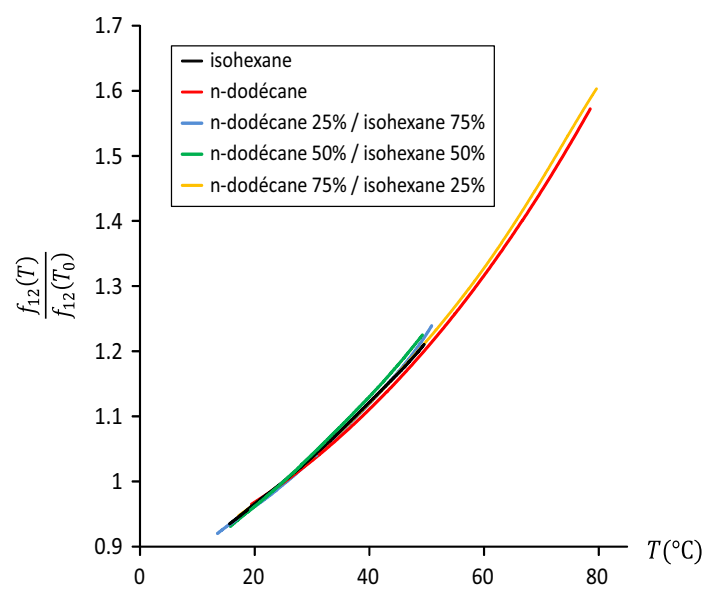

Fig. 6 Calibration curves for $n$-dodecane/isohexane mixtures

has nearly the same dependence in temperature when dissolved into them. For instance, this is the case of isohexane, $n$-decane and $n$-dodecane. Calibrations confirm the almost independence of the temperature response $f_{12}$ on the proportions of these mixtures. An example is presented in Fig. 6 in the case of binary mixtures made of isohexane and $n$-dodecane. In the worst-case scenario (use of a unique expression for $f_{12}$ whatever the initial composition of the mixture, total evaporation of isohexane during the measurements), the measurement error associated with the calibration in temperature will not exceed $2{ }^{\circ} \mathrm{C}$ if the temperature remains in the range of the calibration between 20 and $80^{\circ} \mathrm{C}$. This error compares to the measurement uncertainties when doing the calibration (about $1{ }^{\circ} \mathrm{C}$ for the liquid temperature measurements and $1 \%$ for the fluorescence ratio $R_{12}$ ). The influence of the composition is more important when the liquid fuel contains ethanol or $n$-heptane as illustrated in Fig. 7 for $n$-heptane/ $n$-dodecane mixtures. The calibration function $f_{12}$ is weakly affected by the composition for volume fractions of $n$-heptane ranging between 0 and $75 \%$. Below $25 \%$ of

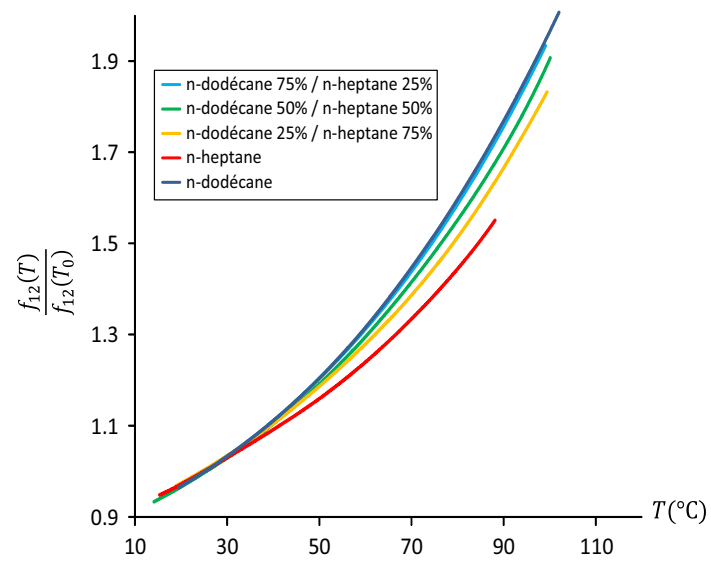

Fig. 7 Calibration curves for $n$-dodecane/ $n$-heptane mixtures

$n$-heptane, composition has practically no effect. In practice, measurement errors can be limited if the calibration in temperature associated with the initial composition of the droplets is used. However, it is necessary to verify that the change in composition remains sufficiently small to neglect its effects on the measurements. Presently, numerical simulations based on the isolated droplet indicate that the change in the drop composition is rather limited during the time period of the measurements (roughly $10 \mathrm{~ms}$ ).

In the expression of the fluorescence signal (Eq. 1), $K_{\text {spec }, i}(\lambda)$ relates to the absorption cross section and the fluorescence quantum yield at a temperature arbitrary chosen to have $f_{i}(\lambda)=1$. This term is therefore likely to depend on the liquid fuel composition. This is confirmed by comparing emission spectra recorded at a fixed temperature of $25{ }^{\circ} \mathrm{C}$ for several isohexane/ $n$-dodecane mixtures. In Fig. 8, noticeable changes in the fluorescence intensity can be pointed out in the yellow-green region of the emission by pyrromethene 597-C8. Unfortunately, this spectral region coincides with one of the two detection bands retained for measuring the liquid temperature. The influence of the fuel composition on the fluorescence ratio $R_{12}$ was investigated more thoroughly for several mixtures of fuels made of alkanes. In Fig. 9, significant variations of the ratio $R_{12}$ are observed when changing the fuel composition at a fixed temperature. For example, in the case of isohexane $/ n$-dodecane mixtures, $R_{12}$ varies from 0.5 to 0.8 at a fixed temperature of $25^{\circ} \mathrm{C}$. Also, the fuel composition seems to have a more pronounced influence on the fluorescence ratio, when the species that compose the mixture have very different molecular weights such as isohexane $/ n$ dodecane or iso-hexane $/ n$-hexadecane. If nothing is done to take into account the effect of the fuel composition, significant errors in the temperature measurements may be committed. Maqua et al. (2006) did a correction for Rhodamine $\mathrm{B}$ dissolved in ethanol/acetone mixtures, which consists in 


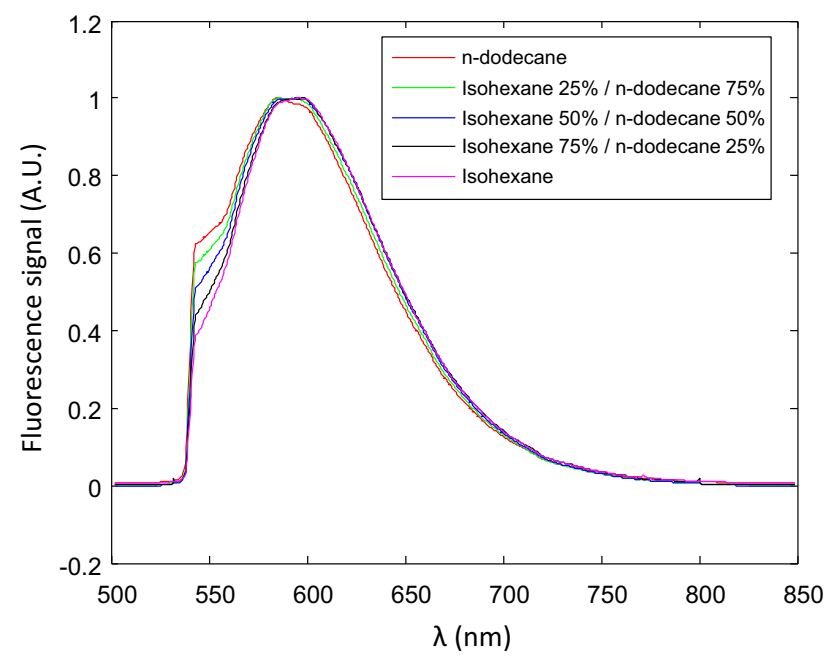

Fig. 8 Emission spectra of pyrromethene 597-C8 at $T=25{ }^{\circ} \mathrm{C}$ in several isohexane $/ n$-dodecane mixtures

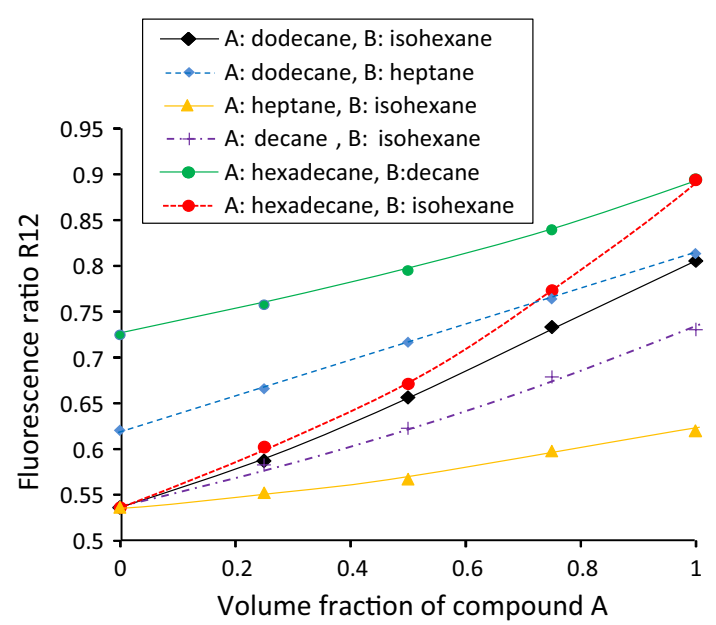

Fig. 9 Evolution of the fluorescence ratio $R_{12}$ as a function of the volume fraction of several liquid fuels mixed at $25{ }^{\circ} \mathrm{C}$. Spectral bands of detection are those displayed in Fig. 3

detecting the signal on a third spectral band having intermediate sensitivities to both composition and temperature. Instead, the change in droplet composition will be estimated from the reduction in the droplet size in the case of binary mixtures made of fuels having very different volatilities. For liquid fuels having about the same volatility, corrections are less necessary since the composition changes at slower rate and with a more limited influence on the fluorescence ratio.

\subsection{Effect of morphology-dependent resonances}

Some difficulties may be encountered when implementing the 2 cLIF technique to droplets. One of these difficulties

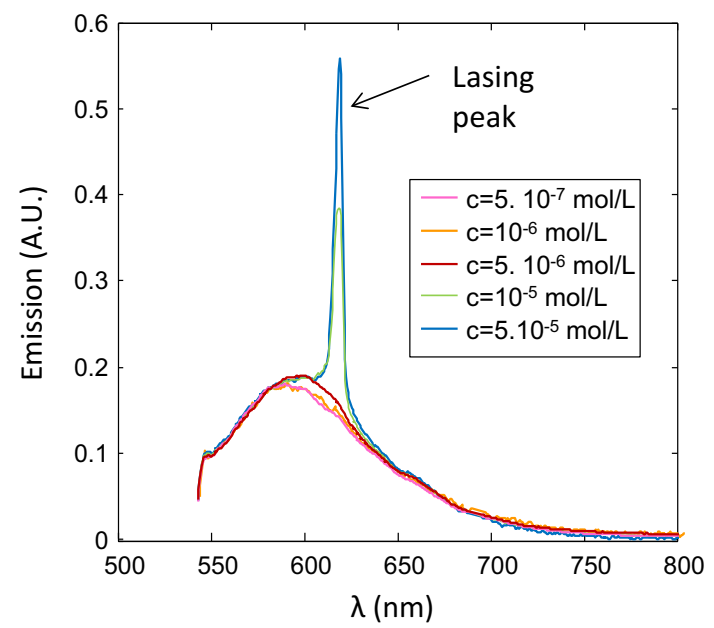

Fig. 10 Emission spectra recorded on $100-\mu \mathrm{m}$ decane droplets with several concentrations in pyrromethene 597-C8

relates to the coupling of the fluorescence emission of the dye and MDRs also termed whispering gallery modes (WGMs). In the presence of a fluorescent dye, stimulated emission of the fluorescent dye can occur inside the spherical drops for high-Q gallery WGMs that coincide with an emission band of the dye. Figure 10 shows emission spectra recorded from $n$-decane droplets sizing about $100 \mu \mathrm{m}$ and seeded with pyrromethene 597-C8 at concentrations ranging from $10^{-7}$ to $5 \times 10^{-5} \mathrm{~mol} / \mathrm{L}$. Integration times of several seconds were required to accumulate sufficient light from the droplets. Each spectrum in Fig. 10 is therefore the contribution of thousands of droplets. A peak due to lasing inside the droplets is clearly visible near $620 \mathrm{~nm}$ at the highest concentrations in fluorescent dye. This peak is relatively large (about $10 \mathrm{~nm}$ ) as it corresponds to a set of several MDRs occurring in tens of thousands droplets. A careful observation of Figs. 10 and 11 indicates that the position of the lasing peak corresponds to a spectral region where selfabsorption is low and light emission by the fluorescent dye is high. Outside the overlap region between the absorption and emission spectra, since self-absorption is very weak, increasing the concentration in fluorescent dye is in favor of more light amplification and enhanced stimulated emission. This explains why the lasing peak is strengthened with an increase in the dye concentration in Fig. 10.

Unfortunately, the lasing of the fluorescent dye inside the droplets is particularly detrimental to temperature measurements as the lasing wavelengths coincide with one of the detection spectral bands. Also in Fig. 12, a noticeable scattering of $R_{12}$ can be observed due to the presence of MDRs. Variations of $R_{12}$ are certainly due to slight deviations in the droplet sphericity. However, the direct observation of the droplets shows hardly some evidence of droplet deformations, which in turn confirms that MDRs are extremely 
sensitive to the morphology. To eliminate the adverse effect of lasing due to MDRs, a non-fluorescent dye, Oil Blue N (also named solvent Blue 14, CAS no. 2646-15-3), is added to the liquid fuel. This dye is miscible in the alkanes and

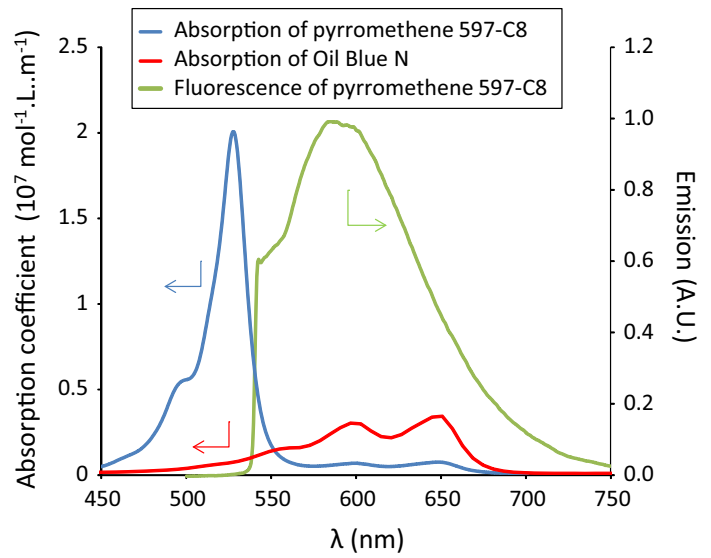

Fig. 11 Absorption and emission spectra of pyrromethene 597-C8 and Oil Blue N

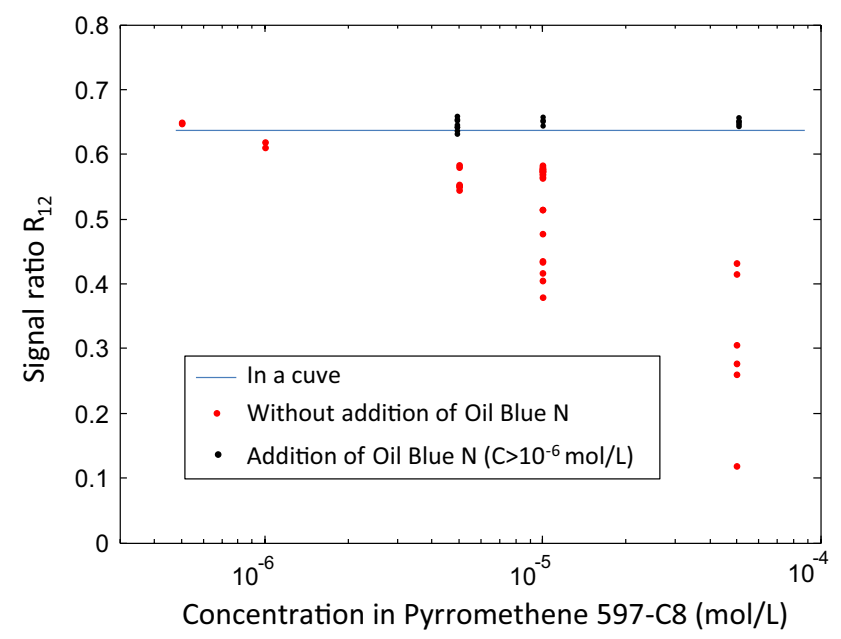

Fig. 12 Influence of the addition of Oil Blue $\mathrm{N}$ the alcohols. Its band of absorption covers the spectral regions where absorption by pyrromethene $597-\mathrm{C} 8$ is too weak to prevent light amplification (Fig. 11). A concentration in Oil Blue $\mathrm{N}$ as low as $10^{-6} \mathrm{~mol} / \mathrm{L}$ was sufficient to recover the same value of the fluorescence ratio $R_{12}$ than in a cell without any morphological resonances (Fig. 12).

\subsection{Effect of the measurement volume}

Due to the lens effect induced by the curvature of the droplet surface and the potential absorption losses inside the liquid medium, the excitation field of the fluorescence is not uniform within the droplets, which means that fluorescence is emitted by different points with different probabilities of reaching the detector. This results in an uneven weighting of the temperature measured by the LIF system. To quantify this effect, it is necessary to take into account the characteristics of the optical system, in particular the sizes of the excitation beam and the detection field of view with respect to the droplet diameter. Presently, the excitation volume is formed by the intersection of two laser beams whose diameter at the beam waist is about $136 \mu \mathrm{m}$. The zone visualized by the optical fiber through the lens doublet of the detection optics corresponds to a disk of about $140 \mu \mathrm{m}$. Geometrical optics is used in association with ray tracing to describe the propagation of light inside the droplet and to evaluate the signal collected by the detection optics. Calculations are illustrated in Fig. 13 for two realistic cases taken from the experiments. To simulate the evolution of the temperature field inside the droplet, the effective thermal conductivity (ETC) model introduced by Abramzon and Sirignano (1989) was used. In this model, the temperature field is considered to have a purely radial symmetry and the thermal conductivity of the liquid is increased to account for heat advection by the internal circulation (Hill vortices). In Fig. 13, $T_{\mathrm{c}}, T_{\mathrm{s}}$ and $T_{\mathrm{m}}$ denote, respectively, the temperature at the droplet center, the surface temperature and the arithmetic mean temperature averaged over the whole droplet volume. The distribution of fluorescence inside the droplet is evaluated from Eq. 1 using
Fig. 13 Comparison between the temperature at the droplet center $T_{\mathrm{C}}$, the surface temperature $T_{\mathrm{S}}$, the arithmetic mean temperature $T_{\mathrm{m}}$ and the temperature $T_{\mathrm{LIF}}$ predicted for the 2cLIF measurements. Calculations correspond to the simulation of the experimental cases in Fig. 23. a Ethanol, b $n$-dodecane
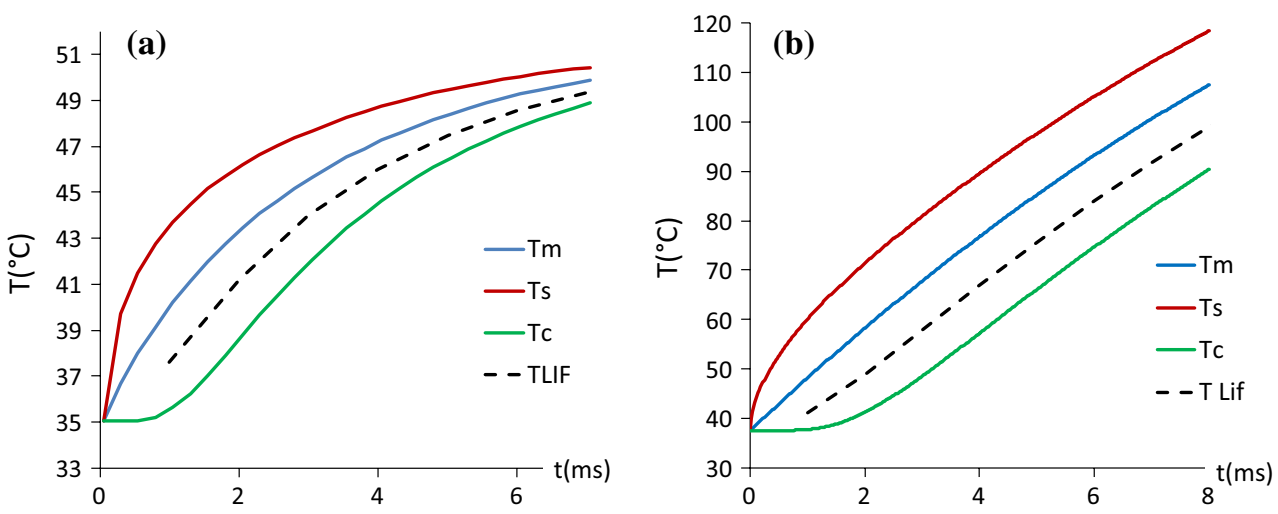
the excitation field provided by ray tracing and the temperature profile calculated from the ETC model. Ray tracing is then used a second time to determine the signal collected by the detection system. Figure 14 is an image of the fluorescence field as it is viewed by the detection optics in the case of a droplet that is slightly off-centered with respect to the laser beams and the detection system. Calculations are repeated for different positions of the droplet to mimic the signal variation due the drop displacement relatively to the detection optics and excitation beams (Fig. 15). The fluorescence signal is integrated over the time period corresponding to the crossing of the measurement volume by the droplet,

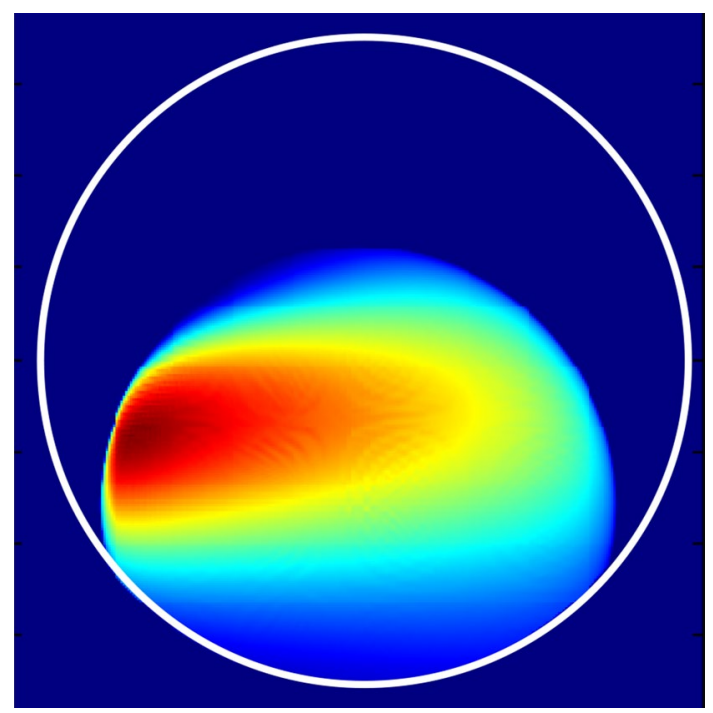

Fig. 14 Fluorescence field viewed by the detection system. Case of a $112-\mu \mathrm{m}$ droplet shifted vertically by $40 \mu \mathrm{m}$ with respect to the measurement volume

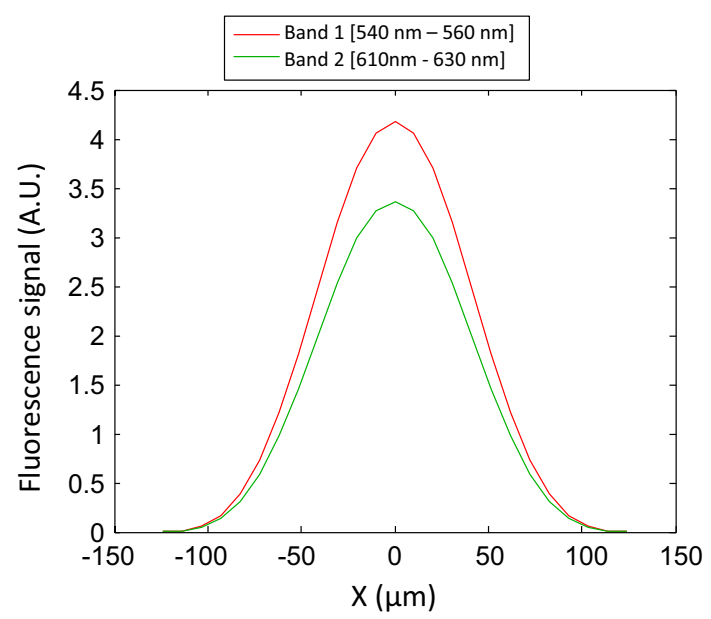

Fig. 15 Evolution of the fluorescence signal as a function of the vertical position of the droplet within the measurement volume $(X=0$ corresponds to a droplet centered in the measurement volume)

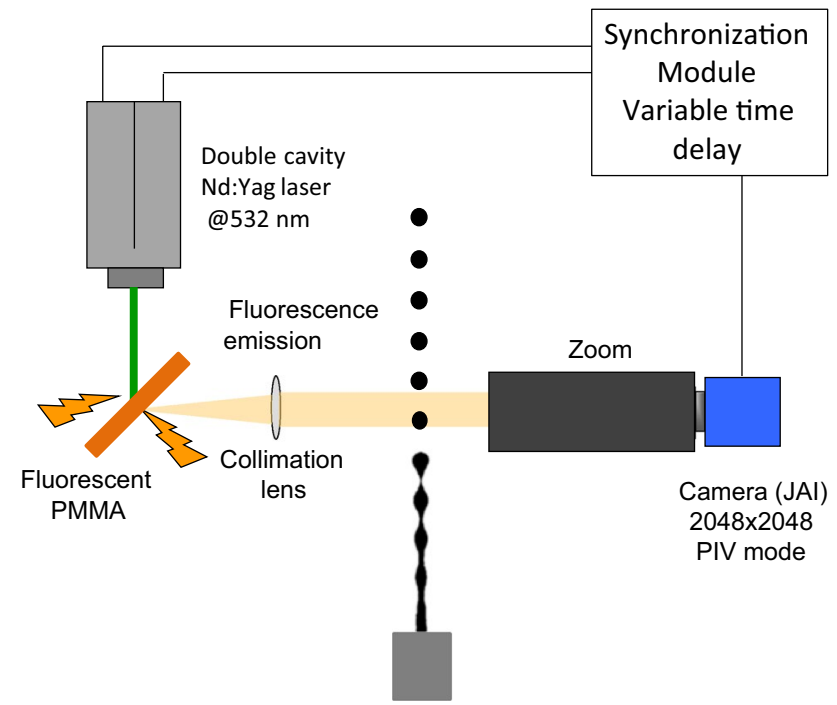

Fig. 16 Experimental setup for the measurement of the droplet diameter by shadowgraphy

and the fluorescence ratio is converted into temperature exactly the same manner as the signal is processed in the experiments. The resulting temperature is denoted $T_{\mathrm{LIF}}$. Figure 13 shows that $T_{\mathrm{LIF}}$ can differ from the arithmetic mean temperature by a few degrees. Obviously, the gap between $T_{\mathrm{LIF}}$ and $T_{\mathrm{m}}$ is all the more important that temperature gradients are strong inside the droplet. The differences between the two temperatures vanish when $T_{\mathrm{s}}$ and $T_{\mathrm{c}}$ are close. In the heating phase, it is observed that $T_{\mathrm{LIF}}$ always underestimates the mean temperature, which indicates that the central region of the droplet has a more important weight on the temperature measured by LIF. For the measurements presented in Fig. 13, it can be roughly estimated that $T_{\mathrm{LIF}} \approx\left(T_{\mathrm{c}}+T_{\mathrm{m}}\right) / 2$. This estimate is probably acceptable for other experimental conditions given that the Peclet number and thus the effective thermal conductivity significantly differ for the two cases presented in Fig. 13 (the Peclet number is about 200 for the ethanol case and 40 for the $n$-dodecane case).

\subsection{Droplet size and velocity measurements}

The size and the velocity of the droplets were measured by quantitative shadowgraphy. A double cavity PIV laser was focused on a solid fluorescent PMMA material. The resulting fluorescent spot is placed at the focal length of a convergent lens to produce an expanded beam of fluorescence light (Fig. 16). This beam is used as a backlit source to observe the droplets by shadowgraphy. The laser pulses duration is about $5 \mathrm{~ns}$, and the fluorescence lifetime of the fluorescent PMMA is also on the order of a few nanoseconds. Hence, motion blur remains insignificant whatever the magnification and the integration time of the 


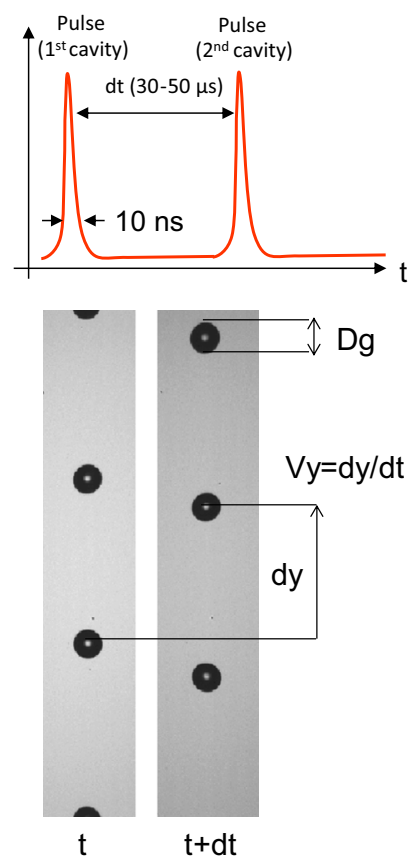

Fig. 17 Principle of the measurement of the droplet diameter and velocity

visualization system. Also, this method has the advantage to avoid some drawbacks inherent to coherent light such as speckles. The signal used for triggering the injection of the droplets is employed to synchronize the flashes of the laser cavities. The frequency of the laser shots is fixed at a few $\mathrm{Hz}$, while the number of droplets injected per second is in the order of $10 \mathrm{kHz}$. Shadow images are recorded by a CCD camera $(2,048 \times 2,048)$ in PIV mode. This camera is equipped with a zoom (OPTEM $125 \mathrm{c}$ with a $2 \times$ magnification module), which allows a $0.3 \mu \mathrm{m} / \mathrm{pxl}$ resolution at the maximum magnification. In practice, the selected magnification results from a compromise between the accuracy of the size measurements and that of the velocity measurements. Magnification of the zoom lens is adjusted in such a way that the motion of 2 or 3 drops can be observed in the pair of images as shown in Fig. 17. Usually this leads to a spatial resolution of about $1 \mu \mathrm{m} / \mathrm{pxl}$.

Image processing begins with a correction of the optical aberrations, which can be important at the high magnifications by the zoom lens. A grid of periodically spaced dots is used for this correction. A spatial transformation is inferred from the coordinates of the dot centers, and the magnification factor is deduced knowing the distance between the dots of the grid. The pincushion distortion does not affect the points near the center of the image. As the grid is regular, it is thus possible to estimate what would be the position of any dot in the absence of any distortion. Denoting $\left(x_{i}, y_{i}\right)$ the coordinates of the $i$ th dot in the image of the grid and $\left(u_{i}, v_{i}\right)$ its corrected coordinates without distortion,

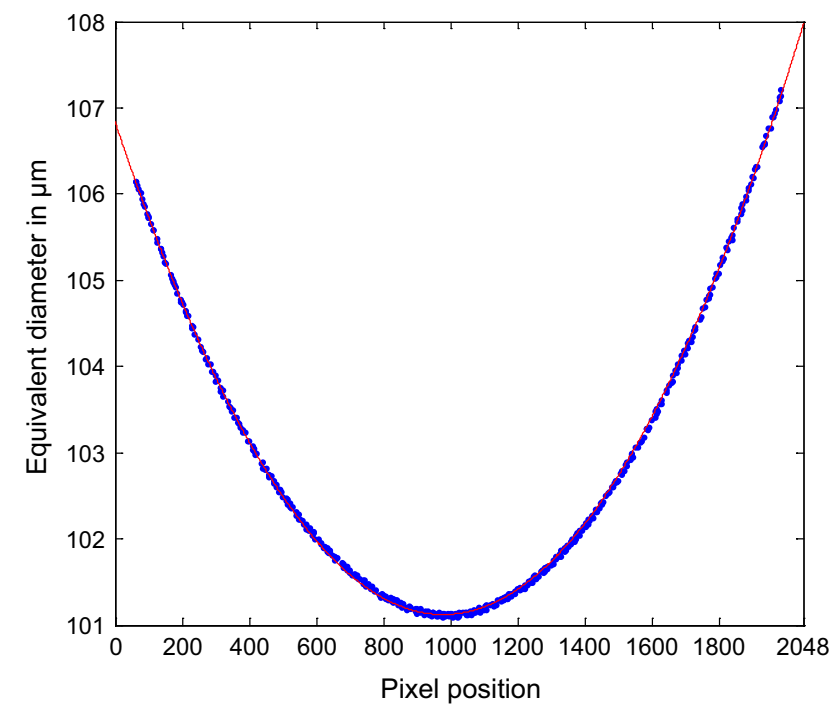

Fig. 18 Droplet size measurements as a function of the vertical position in the images given in pixels. Sizes are obtained without any correction of the image distortion (but using a calibration target to have the magnification factor). In red, a second-order polynomial fitted line that can be used to correct the pincushion distortion by assuming that the droplets are in focus in the images during the measurements

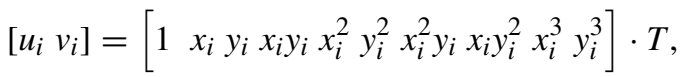

where the matrix $T$ has a size 10-by-2. The elements of $T$ are determined using the least mean square method. Finally, the third-order polynomial transformation defined by $T$ in Eq. 4 is applied to all of the pixels in the image. The difficulty with this approach is the positioning of the dotted target in the calibrations and the droplet streams in the measurements, which must be perfectly perpendicular to the optical axis and in the focal plane. In practice, an alternative method to evaluate the droplet size relies on the periodicity of the droplets. Figure 18 shows the size of the droplets as a function of the vertical position in the images without any correction of the pincushion distortion. The effect of the pincushion distortion can be clearly pointed out and a second-order polynomial seems satisfactory to fit the data points. The equivalent diameter based on the surface area of the droplet reaches a minimum at the center of the image, which agrees well with a pincushion distortion. Given that the pincushion distortion does not affect the center of the images, the actual size of the droplets can be well determined by assuming that it corresponds to the minimum of the second-order polynomial fitting the experimental data. The distances of the measurement points to the interpolating polynomial are related to the size dispersion of the droplets that is usually very small in the experiments as illustrated in Fig. 18.

A grayscale threshold is applied to identify the droplets in the grayscale images. The main difficulty is due to 
Fig. 19 Evolution of the drop size and the drop velocity ( $n$-dodecane and ethanol droplet streams injected in air at $24{ }^{\circ} \mathrm{C}$ under isothermal conditions, and $d_{0}=113.4 \mu \mathrm{m}$ for ethanol) $d_{0}=101.2 \mu \mathrm{m}$ for $n$-dodecane
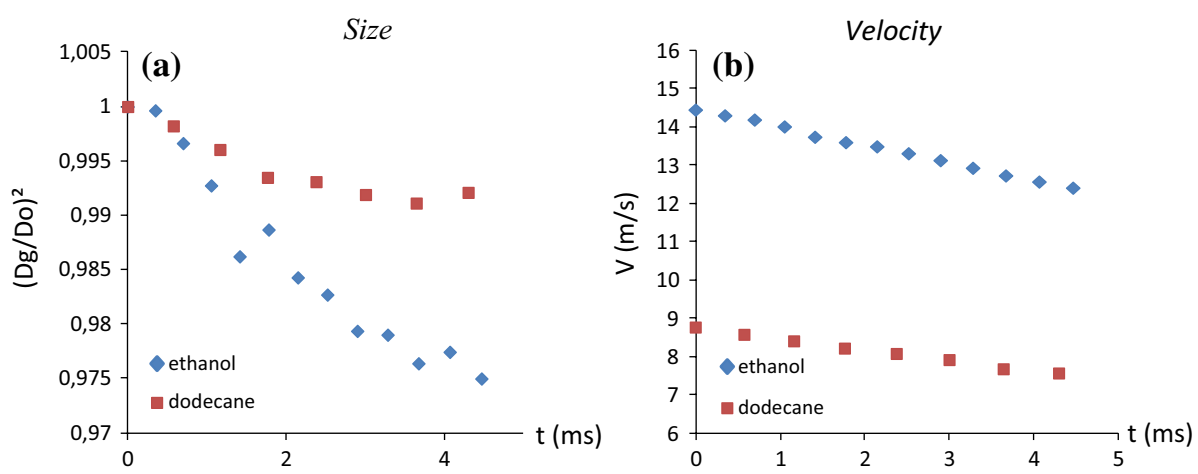

Fig. 20 Distributions of size and velocity measurements obtained at $t=0 \mathrm{~ms}$ in the case of the $n$-dodecane droplet stream presented in Fig. 19 (a) Size $(d=101.16 \mu \mathrm{m}$ RMS $=0.03 \mu \mathrm{m})$

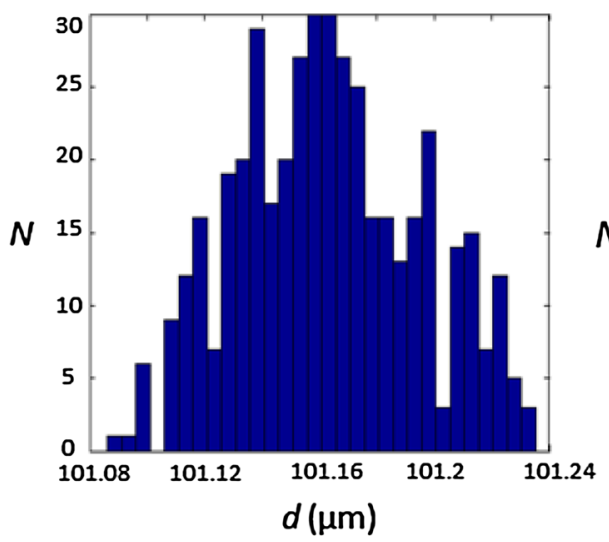

(b) Velocity $\left(V=8.77 m \cdot \mathrm{s}^{-1} \mathrm{RMS}=0.07 \mathrm{~m} / \mathrm{s}\right)$

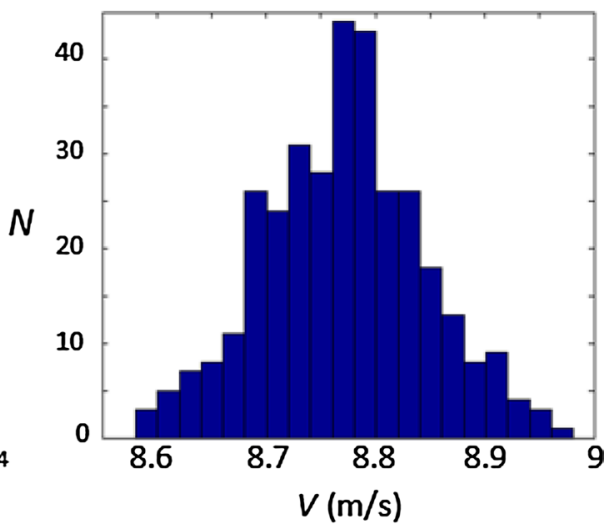

the non-uniformity of the background. Gray levels of the background may also vary because of the limited shotto-shot stability of the laser flashes. A background image is obtained by averaging several images taken without any droplet. Images of the droplets are then divided by this background image and normalized by scaling them between 0 and 1 . The threshold for the segmentation of the images is set at a value of 0.5. Finally, ellipses that fit to the points of the drop contours are interpolated. The center of the fitting ellipse is considered as the droplet center, while the equivalent diameter is based on its surface area. It should be emphasized that the droplets are perfectly spherical in the measurements that are reported in this study. Droplets may appear as ellipses in the images because of the optical aberrations that distort the droplet shape. Otherwise, surface oscillations are completely damped given that the measurements are taken at several centimeters from the injector outlet. The determination of the velocity can be easily performed provided that there is no ambiguity to associate the droplets in the pairs of images. For that, the delay time $\mathrm{d} t$ between the shots of the two laser cavities has to be carefully adjusted. The value of $\mathrm{d} t$ is generally fixed to the time period of the drop injection (about $30 \mu \mathrm{s}$ ). Indeed, there is no ambiguity with this time period since a droplet will be located at $t_{0}+\mathrm{d} t$ near the position of the droplet that was ahead of it at time $t_{0}$ (Fig. 17).

The absolute accuracy of the drop sizing is directly related to the spatial resolution, i.e., about $1 \mu \mathrm{m}$, but the relative accuracy within a set of measurements can be much better if the images are recorded with the same zoom factor and the same illumination. To measure accurately the size of the droplets, it is also critical to properly do the focus on the droplets. A defect of focus is actually the main source of errors in the measurements. To assess how accurate measurements can be, the method was tested on ethanol and $n$-dodecane droplets injected at room temperature as illustrated in Figs. 19 and 20. The acquisitions consist in 100 pairs of images, which proves largely sufficient to insure the statistical convergence of the data with a RMS as low as $0.03 \mu \mathrm{m}$ for the size and $0.07 \mathrm{~m} / \mathrm{s}$ for the velocity (Fig. 20). Very small decreases in the drop size (smaller than $1 \mu \mathrm{m}$ ), caused by evaporation, can be effectively captured as shown in Fig. 19.

\section{Measurement results and discussion}

The previously described techniques were used to study the heating and the evaporation of droplets made of different fuels. The measurements reported herein were obtained in 
dry air at $540{ }^{\circ} \mathrm{C}$. Many parameters are known to influence the droplet evaporation. Quantifying the effect of these parameters can be rather challenging. From an experimental point of view, it was difficult with the present injection system to change independently the size, the velocity and the droplet spacing at the injection.

\subsection{Effects of fuel volatility}

Different behaviors were observed from the study of liquids having different volatilities. Figure 21 allows comparing the evaporation of different fuels including ethanol, $n$-heptane, $n$-decane and $n$-dodecane. This figure displays the evolutions of the temperature and size of droplets that are injected in rather similar conditions, except for the $n$-heptane droplets stream that has substantially higher velocities and lower initial temperature.

For high-volatility fuels such as isohexane and ethanol, the droplet temperature reaches rapidly equilibrium at a value that is much lower than the boiling temperature of the fuel. For instance, this temperature is about $40{ }^{\circ} \mathrm{C}$ for isohexane and $50{ }^{\circ} \mathrm{C}$ for ethanol, while the boiling points of these liquids are, respectively, 60 and $78{ }^{\circ} \mathrm{C}$. In fact, this equilibrium temperature corresponds to the wet-bulb temperature of the fuel in the hot environment of the chamber. When the thermal equilibrium is reached, the heat transfer to the droplet is entirely used for liquid vaporization. In Fig. 21, the size of isohexane, ethanol and $n$-heptane droplets appears to follow the $d^{2}$ law (i.e., the drop surface decreases linearly with time) after they nearly reach the equilibrium temperature. The time required for that is very short for isohexane that is very volatile. For less volatile fuels, such as $n$-decane and $n$-dodecane, the liquid temperature must be relatively high for the evaporation to be significant. In Fig. 21, $n$-decane and $n$-dodecane droplets simply do not have a sufficient time to reach such a high temperature. As a consequence, the droplet size increases because of the thermal expansion of the liquid that compensates the size reduction due to evaporation. Basically, all the heat transferred to droplets made of low-volatile fuels is used for heating up the liquid. This explains why $n$-heptane, $n$-decane and $n$-dodecane droplets have nearly the same heating rate until evaporation starts being significant for $n$-heptane and $n$-decane after $t=2 \mathrm{~ms}$. By increasing the injection temperature of the fuel, it was possible to determine that the equilibrium temperature is about $170{ }^{\circ} \mathrm{C}$ for $n$-dodecane (Fig. 22). When this temperature is reached by the droplets, the evaporation rate is much higher. In fact, the evaporation rate (time derivative of $d^{2}$ ) of $n$-dodecane at the thermal equilibrium is comparable to that of isohexane droplets, provided that similar flow conditions in terms of Reynolds number and drop spacing are achieved.

\subsection{Effects of drop interspace distance on heat and mass transfer coefficients}

To put forward the influence of droplet interactions, it was necessary to compare the measurements to the case of the

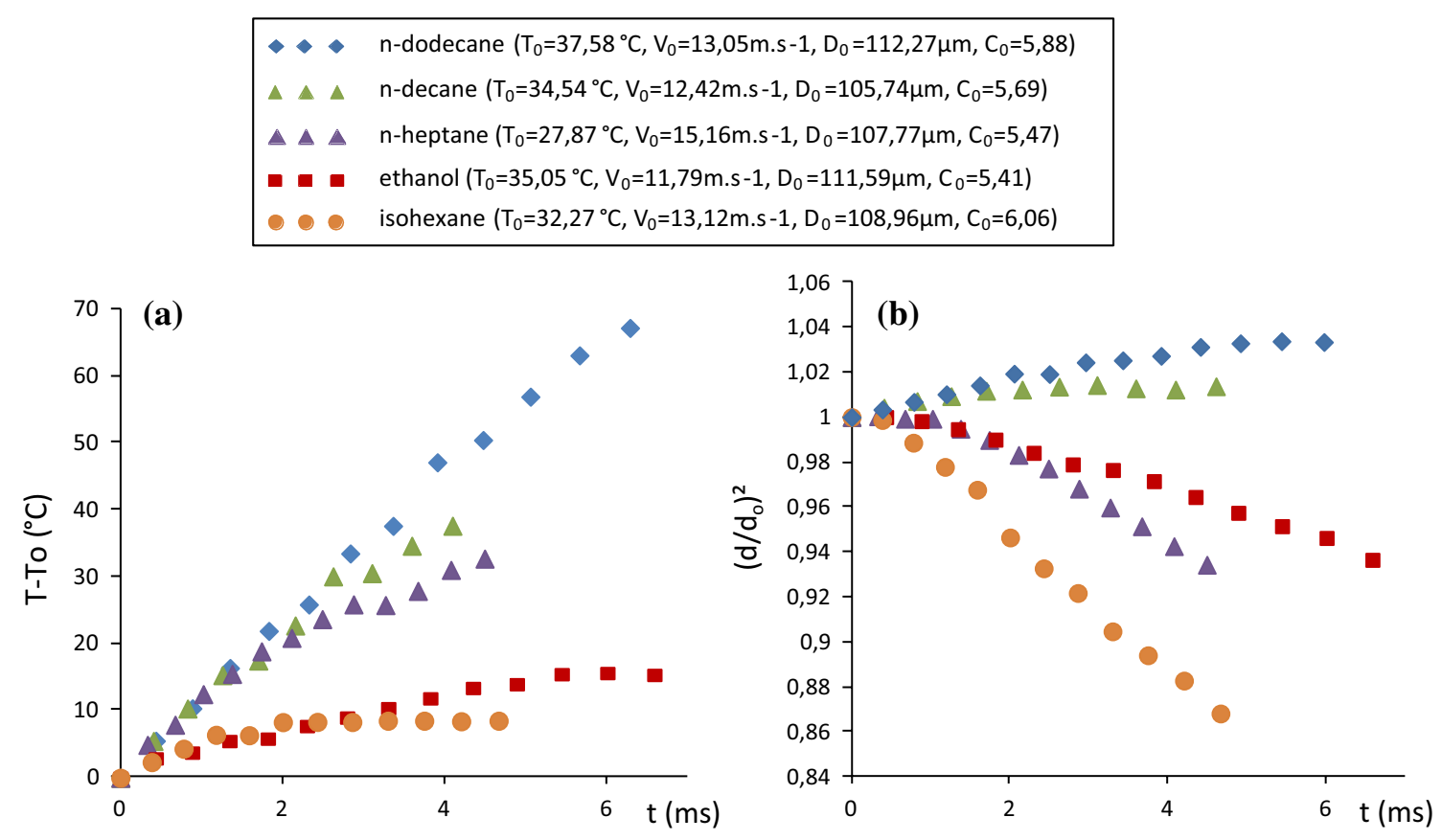

Fig. 21 Evaporation and heating of droplets made of different liquids 


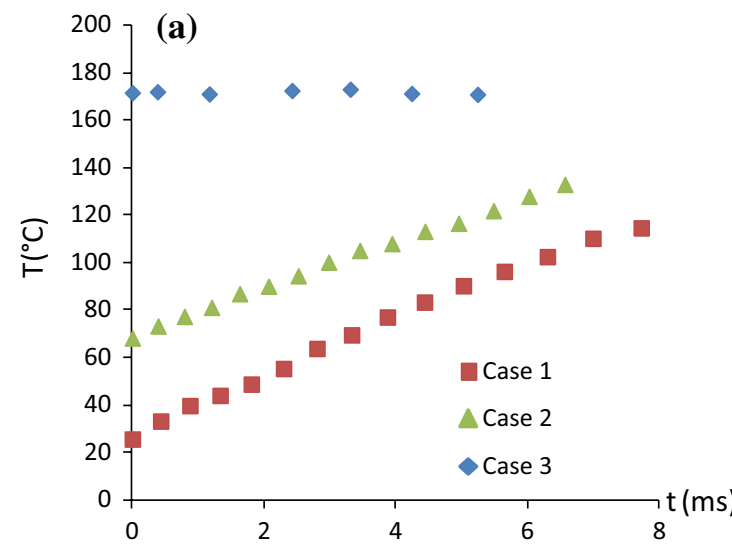

Fig. 22 Evaporation of $n$-dodecane droplets for different injection temperatures. Evolution of the temperature (a), the size (b) (case 1: $T_{0}=26{ }^{\circ} \mathrm{C}, d_{0}=97.4 \mu \mathrm{m}, V_{0}=11.8 \mathrm{~m} / \mathrm{s}, C_{0}=4.2$;

isolated droplet, whose evaporative behavior is known from existing models (Abramzon and Sirignano 1989). Nusselt and Sherwood numbers, denoted $\mathrm{Nu}$ and $\mathrm{Sh}$, respectively, are used to characterize the influence of forced convection on the rate of heat and mass transfers. When a drop is placed into a hot gas environment, it receives a heat flux $\Phi_{\mathrm{C}}$ from the gas and starts to evaporate with an instantaneous mass vapor flowrate $\dot{m}$. Basically, $\Phi_{\mathrm{C}}$ and $\dot{m}$ can be expressed as

$\dot{m}=\pi d \rho_{\mathrm{g}} D_{\mathrm{v}} \operatorname{Sh} B_{\mathrm{M}}$,

$\Phi_{C}=\pi d N u \lambda_{\mathrm{g}}\left(T_{\infty}-T_{\mathrm{s}}\right)$

where $\rho_{\mathrm{g}}$ and $\lambda_{\mathrm{g}}$, respectively, are the density and the thermal conductivity of the gas and $D_{\mathrm{v}}$ is the diffusion coefficient for vapor in air. $B_{\mathrm{M}}=Y_{\mathrm{S}} / 1-Y_{\mathrm{S}}$ is the mass transfer Spalding number related to the mass fraction of vapor $Y_{\mathrm{S}}$ at the drop surface, $T_{\infty}$ is the temperature of the ambient gas, and $T_{\mathrm{S}}$ is the droplet surface temperature. In the case of the isolated droplet, the values of Nusselt and Sherwood numbers (respectively $N u_{\text {iso }}$ and $S h_{\text {iso }}$ ) can be determined from the film theory and the quasi-steady description of droplet evaporation (Abramzon and Sirignano 1989),

$N u_{\text {iso }}=\left(2+0.6 \operatorname{Re}^{0.5} \operatorname{Pr}^{1 / 3} / F_{\mathrm{T}}\right) \ln \left(1+B_{\mathrm{T}}\right) / B_{\mathrm{T}}$

$S h_{\text {iso }}=\left(2+0.6 R e^{0.5} S c^{1 / 3} / F_{\mathrm{M}}\right) \ln \left(1+B_{\mathrm{M}}\right) / B_{\mathrm{M}}$

where $F=(1+B)^{0.7} \ln (1+B) / \ln (1+B) B \cdot B, F_{\mathrm{M}}=F\left(B_{\mathrm{M}}\right)$, $F_{\mathrm{T}}=F\left(B_{\mathrm{T}}\right), B$ being equal to $B_{\mathrm{M}}$ or $B_{\mathrm{T}}$.

These expressions of $N u_{\text {iso }}$ and $S h_{\text {iso }}$ were used to simulate the isolated droplet heating and evaporation following the numerical procedure described by Abramzon and Sirignano (1989). However, in the presence of drop interactions,

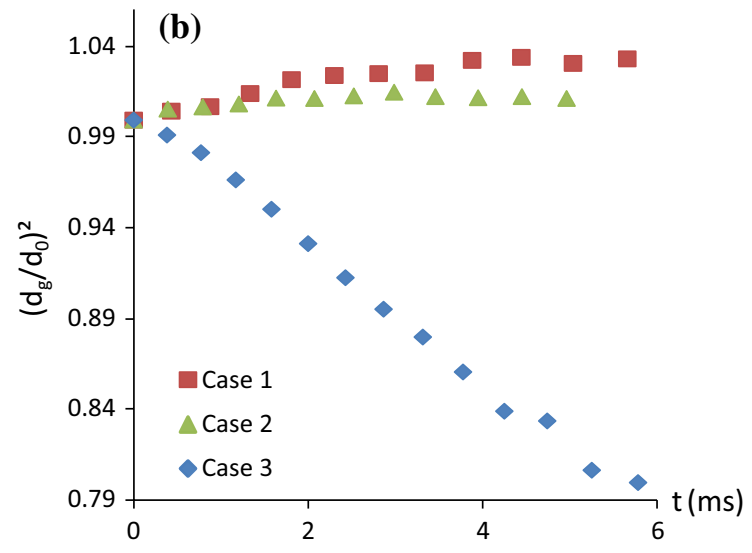

case 2: $T_{0}=68{ }^{\circ} \mathrm{C}, d_{0}=99.1 \mu \mathrm{m}, V_{0}=13 \mathrm{~m} / \mathrm{s}, C_{0}=4.5$; case 3: $\left.T_{0}=170{ }^{\circ} \mathrm{C}, d_{0}=92.1 \mu \mathrm{m}, V_{0}=13.3 \mathrm{~m} / \mathrm{s}, C_{0}=4.8\right)$

the heat and mass transfers are known to be reduced. To account for this effect, the reduction factors $\eta_{\mathrm{T}}$ and $\eta_{\mathrm{M}}$ are introduced:

$\eta_{T}=N u / N u_{\text {iso }}$ and $\quad \eta_{\mathrm{M}}=S h / S h_{\text {iso }}$

Droplet diameter and temperature evolutions are simulated for several guess values of $\eta_{\mathrm{T}}$ and $\eta_{\mathrm{M}}$. Retained values are those that minimize the differences between the measurements and the simulation. Acceptable comparison with the experiments can be obtained by assuming that $\eta_{\mathrm{T}}$ and $\eta_{\mathrm{M}}$ are not varying with time. Generally, adjusted values of $\eta_{\mathrm{T}}$ and $\eta_{\mathrm{M}}$ were found to be very close. In particular, it was observed that the equilibrium temperature of the droplets is not affected by the spacing between the droplets. This observation confirms that $\eta_{\mathrm{T}}$ and $\eta_{\mathrm{M}}$ are modified to the same extend by the effects of interactions. Indeed, the ratio $\eta_{\mathrm{T}} / \eta_{\mathrm{M}}$ controls the balance between the heat flux of vaporization $\left(L_{\mathrm{v}} \dot{m}\right)$ and the heat flux transfer to the droplets $\Phi_{\mathrm{C}}$ and thus the equilibrium temperature. The sensitivity on the adjustment of $\eta_{\mathrm{M}}$ is poor for low-volatile fuels that have very low evaporation rates like $n$-dodecane injected at room temperature. Figure 23 shows the size and temperature evolutions of two droplet streams made of ethanol and $n$-dodecane, respectively. Only the correction factor $\eta_{\mathrm{T}}$ can be estimated in the case of the $n$-dodecane droplet stream, since the heat transfer to the droplet is almost totally used to increase the liquid temperature. In general, at least one of the two correction coefficients $\eta_{\mathrm{M}}$ or $\eta_{\mathrm{T}}$ can be determined with a reasonable accuracy (of the order of 0.1). It should also be noted that the model of Abramzon and Sirignano, although it is widely used, remains an approximation. This obviously introduces an additional uncertainty in the values of $\eta_{\mathrm{M}}$ and $\eta_{\mathrm{T}}$ that cannot be easily quantified. Nevertheless, even if the absolute values of the reported reduction factors may be affected by large errors, their trend presented hereafter may remain significant. 

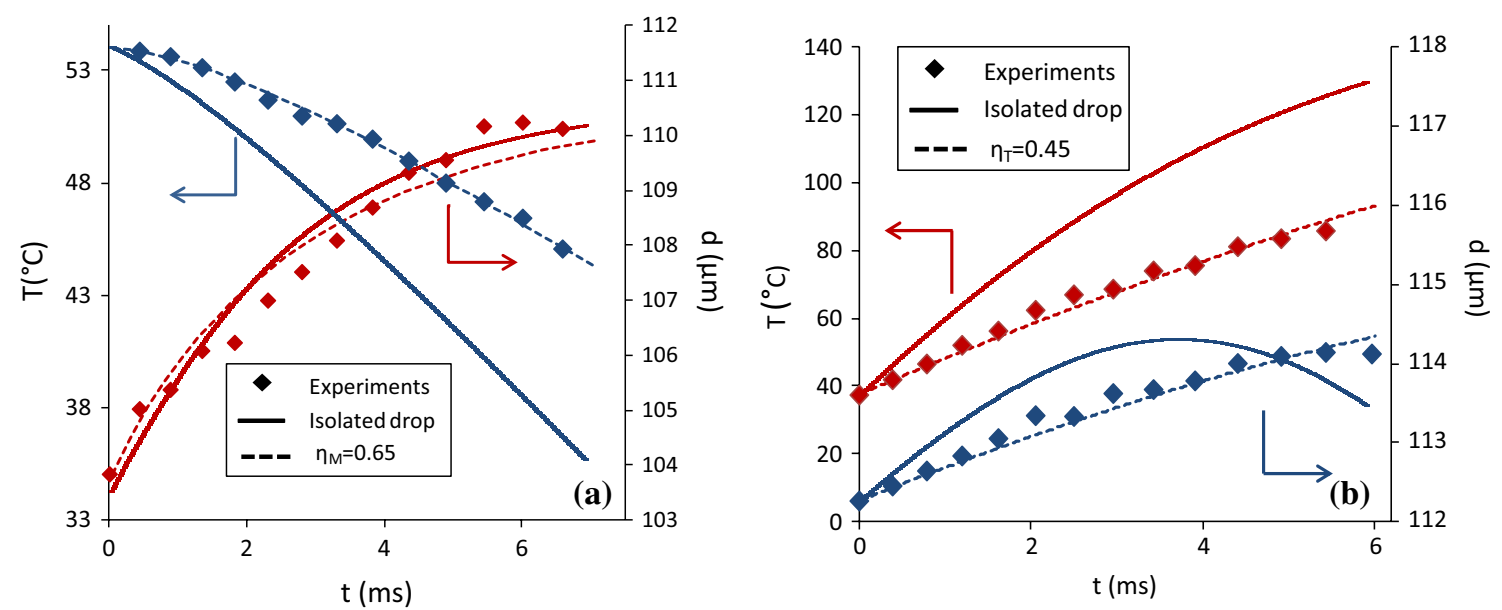

Fig. 23 Evolutions of temperature and size in the case of two droplet streams. a Ethanol $\left(T_{0}=35.1{ }^{\circ} \mathrm{C}, d_{0}=111.6 \mu \mathrm{m}, V_{0}=11.8 \mathrm{~m} / \mathrm{s}\right.$, $\left.C_{0}=5.4\right)$. b $n$-Dodecane $\left(T_{0}=37.6{ }^{\circ} \mathrm{C}, d_{0}=112.3 \mu \mathrm{m}, V_{0}=13.1 \mathrm{~m} / \mathrm{s}, C_{0}=5.9\right)$

In Fig. 23, the dimensionless drop spacing and the Reynolds number are very close for the two droplet streams, but the heat and mass transfers are not reduced to the same extent $\left(\eta_{\mathrm{T}}=0.45\right.$ for $n$-dodecane and $\eta_{\mathrm{M}}=0.65$ for ethanol). To confirm that the fuel nature has an influence on the interactions between the droplets and their consequences on heat and mass transfers, values of $\eta_{\mathrm{T}}$ and $\eta_{\mathrm{M}}$ were estimated for three different fuels (ethanol, $n$-decane and $n$-dodecane) while changing the droplet spacing and the injection temperature for $n$-dodecane. In Fig. $24, \eta_{\mathrm{T}}$ and $\eta_{\mathrm{M}}$ increase with the distance parameter, which was expected. Even for a dimensionless spacing parameter as large as $C=9$, heat and mass transfers are still significantly lower than in the case of the isolated droplet. Moreover, Fig. 24 reveals that the heat and mass transfers are reduced to a large extend in the case of $n$-decane and $n$-dodecane drops injected at $30{ }^{\circ} \mathrm{C}$. The reduction in the heat and mass transfers are lower for ethanol droplets injected at roughly the same temperature $\left(30-50{ }^{\circ} \mathrm{C}\right)$. However, if $n$-dodecane droplets are injected at a temperature close to the equilibrium temperature (roughly $170{ }^{\circ} \mathrm{C}$ ), the reduction in the heat and mass transfer becomes comparable to that observed for the ethanol droplets. This result shows that the volatility of the liquid fuel has a noticeable influence on the interactions between the fuel droplets. Explanations are still needed to understand this phenomenon. Some evidences of an effect of the fuel volatility were already reported by Deprédurand et al. (2010) at much smaller ambient gas temperature and for the same liquid fuels. It can be assumed that the Stefan flow produced by the vapor released by the droplet makes the droplet surface less sensitive to any perturbation in the outer flow that can be induced by the neighbor drops. Estimates of the velocity of the Stefan flow from the experimental evaporation rate $\left(V_{\text {Stefan }}=\dot{m} / \pi \rho_{\mathrm{g}} d^{2}\right)$ give about

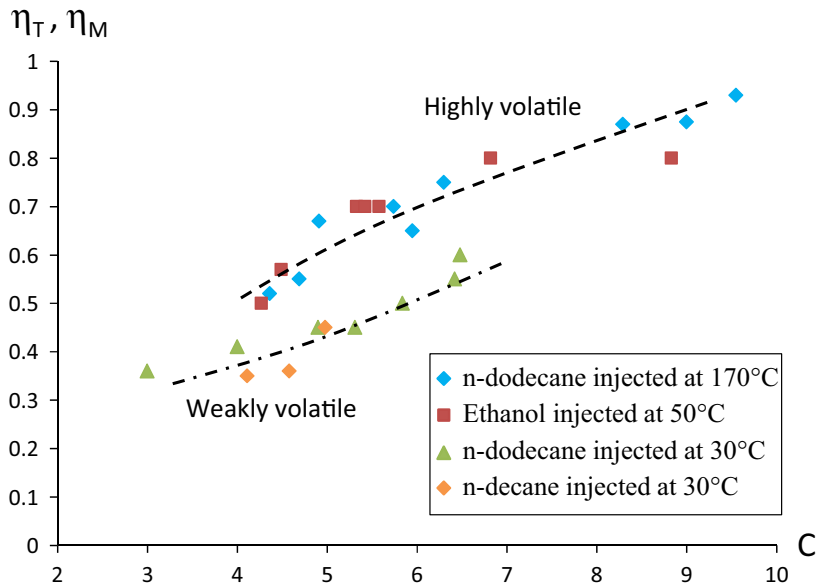

Fig. 24 Influence of the inter-droplet spacing and the fuel volatility on the reduction of the heat and mass transfers

$0.4 \mathrm{~m} / \mathrm{s}$ for ethanol droplets injected at $50{ }^{\circ} \mathrm{C}, 0.6 \mathrm{~m} / \mathrm{s}$ for $n$-dodecane injected at $170{ }^{\circ} \mathrm{C}$ and almost $0 \mathrm{~m} / \mathrm{s}$ when $n$-dodecane is injected at $30{ }^{\circ} \mathrm{C}$. The Stefan flow forms a film around the droplet, which prevents the outer flow to reach directly the drop surface. For a low-volatile liquid fuel, this film does not exist or will take time to form. As a consequence, the flow conditions near the drop surface can be more affected by the modifications of the outer flow.

\subsection{Evaporation measurements in binary droplets}

Finally, the evaporation of multicomponent droplets was also investigated. Figures 25 and 26 display measurements obtained for several mixtures of isohexane and $n$-dodecane. The size, the velocity and the spacing parameter are 


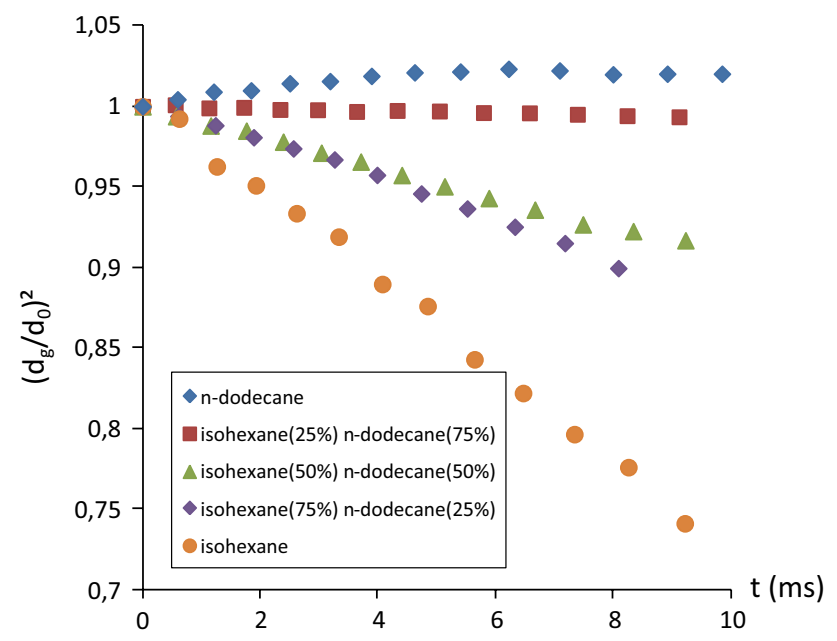

Fig. 25 Evaporation of binary component droplets for similar experimental conditions. Evolution of the droplet size $\left(T_{0}=35^{\circ} \mathrm{C}\right.$, $d_{0}=115 \mu \mathrm{m}, V_{0}=8.5 \mathrm{~m} / \mathrm{s}, C_{0}=6$ )

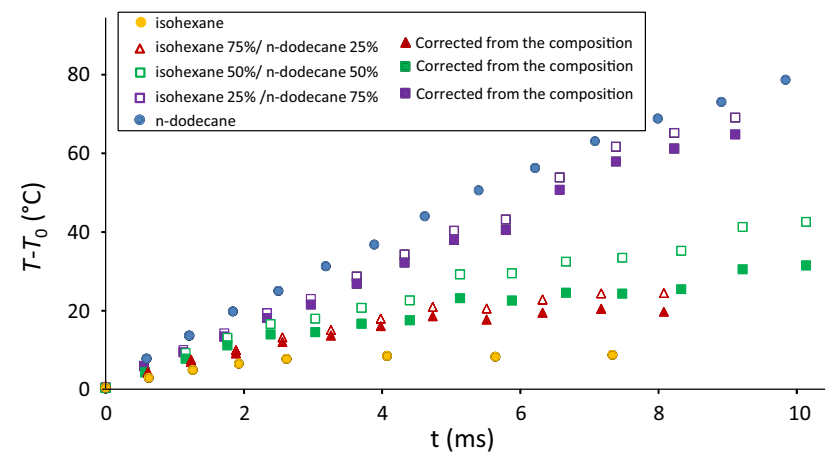

Fig. 26 Evaporation of binary droplets in the same experimental conditions as in Fig. 25. Hollow markers correspond to corrected values of the measurements

roughly the same in these experiments. A noticeable effect of the initial droplet composition can be observed on the droplet heating and the evaporation rate. As expected, increasing the volume fraction of isohexane tends to increase the evaporation rate and to reduce the heating rate. A pseudo-equilibrium temperature can be extrapolated for mixture fractions of $n$-dodecane equal to 25 and $50 \%$. The relative stagnation of the liquid temperature for these two compositions is accompanied by an almost linear variation of the squared diameter. From a qualitative point of view, those mixtures appear to behave as pure compounds. This may be due to the fact that the change in composition remains moderate over the time period of the experiment (Fig. 27). For volume fractions of $n$-dodecane larger than $50 \%$, the temperature rapidly increases and exceeds the boiling temperature of isohexane (about $60{ }^{\circ} \mathrm{C}$ ). However, heating was not rapid enough to observe boiling and



Fig. 27 Evolution of the volume fraction of $n$-dodecane evaluated from Eq. 10 for the same experimental conditions as in Figs. 25 and 26

micro-explosions of the droplets as reported in some comparable works involving mixtures made of heavy and light fuels (Frohn and Roth 2000).

As already mentioned, variations in the droplet composition can affect the temperature measurements. In the case of isohexane $/ n$-dodecane mixtures, a simple assessment of the drop composition can be obtained by assuming that only isohexane evaporates. The conservation of the mass of $n$-dodecane inside the droplet can be written as,

$\rho_{\text {dodecane }}\left(T_{i}\right) V_{i} \frac{\pi d_{i}^{3}}{6}=\rho_{\text {dodecane }}\left(T_{0}\right) V_{0} \frac{\pi d_{0}^{3}}{6}$

In this expression, $V$ denotes the volume fraction of $n$-dodecane inside the droplet and $T$ and $d$ are, respectively, the mean temperature and the size of the droplets. Subscripts 0 and $i$ refer to two distinct instants of the droplet lifetime. In the following, $t_{0}$ is taken at the first measurement point (about $1 \mathrm{~cm}$ after the drop has entered in the chamber). Being not directly measured, the initial volume fraction of $n$-dodecane $V_{0}$ is fixed at its value at the injection. This implies that droplet evaporation is very limited in the period of time between the injection and the first measurement point in the chamber. To find out the droplet composition at the instant $t_{i}$, an iterative procedure is implemented since $V_{i}$ and $T_{i}$ are both a priori unknown in Eq. 10. In a first step, $T_{i}$ is set at the raw measurement value (uncorrected for the change in droplet composition), and 
Eq. 10 is used to estimate the volume fraction $V_{i}$. The correction of the fluorescence ratio for the change in composition is given by,

$R_{12, \text { corr }}=\frac{f\left(V_{0}\right)}{f\left(V_{i}\right)} R_{12}$,

where $f(V)=0.1986 V^{2}+0.1131 V+0.4867$ corresponds to the calibration in composition displayed in Fig. 9. Equation 11 implies that the fluorescence ratio $R_{12}$ is not becoming more or less sensitive to fuel compositions variations when increasing the liquid temperature. This was checked experimentally in the calibration cell for temperature up to $50{ }^{\circ} \mathrm{C}$. The influence of the temperature and the composition can be separated, meaning that it is possible to express the fluorescence ratio as:

$R_{12} \approx \frac{K_{\mathrm{opt}, 1} K_{\mathrm{spec}, 1}}{K_{\mathrm{opt}, 2} K_{\mathrm{spec}, 2}} g(T) \cdot f(V)$

where $f$ and $g$ are two functions that should be determined by calibrations. An updated value of $T_{i}$ is available for a new iteration after conversion of the fluorescence ratio $R_{12 \text {,corr }}$ into temperature. In practice, two or three iterations are sufficient to converge. Figure 27 shows the evolution of the volume fraction of $n$-dodecane obtained by this iterative method. Composition is changing at a faster rate for droplets that initially contains $50 \%$ of $n$-dodecane. Otherwise, the increase in the volume fraction of $n$-dodecane is more limited for the other mixtures. In Fig. 26, temperature measurements are compared with and without the correction for the change in composition. The droplet temperature is increased by a few ${ }^{\circ} \mathrm{C}$, when the change in composition is accounted for. The correction can reach about $8{ }^{\circ} \mathrm{C}$ in the case of the $50 \%$ isohexane $/ n$-dodecane mixture, which is not negligible. The proposed correction has the advantage to be very simple to implement, but is limited to the case where a heavy fuel and a light fuel are mixed. It must be also considered with caution, since the weighing caused by the distribution of the internal excitation field is ignored. As shown, the LIF signal is more sensible to the central region of the droplet, but $T_{i}$ and $V_{i}$ are considered as volume averaged quantities in Eq. 10. Therefore, the previously described correction is based implicitly on the assumption that the composition is uniform inside the droplet, i.e., there is a rapid mixing in the liquid. However, this assumption may not be valid. If a diffusion-limited transfer of mass is considered inside the droplet, composition will change very slowly at the center of the droplet. Presently, the characteristic time of the mass transfer is much longer than the time period covered by the measurements (here about $10 \mathrm{~ms}$ ). The characteristic time of the mass diffusion can be evaluated as $t_{\text {diff }} \sim d^{2} / D_{\text {liq }}$, where the droplet diameter $d$ is about $115 \mu \mathrm{m}$ and the diffusion coefficient $D_{\text {liq }}$ is about $2 \times 10^{-9} \mathrm{~m}^{2} / \mathrm{s}$ for the isohexane $/ n$-dodecane liquid mixture. This gives a time for mass diffusion $t_{\text {diff }} \approx 5 \mathrm{~s}$ that is several orders of magnitude larger than the time covered by the experiments. As long as the composition has not changed significantly in the center of the droplet, doing no correction of the temperature would be acceptable since the center of the droplet is the region with the largest contribution to the LIF signal (it was shown that $T_{\mathrm{LIF}}\left(T_{\mathrm{C}}+T_{\mathrm{m}}\right) / 2$ ). As a result, the measurement method could also apply to any mixtures, provided that mass diffusion remains limited. However, an accurate modeling of the composition and the temperature field within the droplet is required to validate one or the other of these assumptions (rapid mixing with a correction or diffusion-limited transfers with no corrections). The question of the mixing inside the droplet is still an open one, especially in the presence of strong aerodynamic interactions between neighbor droplets that reduce the shear stress at the droplet surface. In the absence of a precise description of the internal mixing process, it can just be said that the actual temperature of the droplets lies between the corrected and uncorrected values of the temperature presented in Fig. 26.

\section{Conclusions}

This study illustrates the capabilities of the 2cLIF thermometry to characterize the temperature of droplets including mono- and multi-component droplets. The fluorescent dopant used in this work, pyrromethene 597-C8, has many advantages for studying the evaporation and the heating of droplets made of alkanes, ethanol and their mixtures. The respective influence of the temperature and the composition appear to be relatively well separated. The sensitivity of the fluorescence ratio to the fuel composition is more pronounced in alkane mixtures made of compounds that have very different molecular weights such as isohexane $/ n$ dodecane mixtures. Otherwise, droplet temperature can be measured ignoring the change in the liquid composition during the droplet evaporation in the conditions encountered in the described experiments. The 2cLIF method has therefore the potential to be generalized to more complex mixtures.

Furthermore, the addition of a small amount of an absorbing but non-fluorescent dye, such as Oil Blue N, allows avoiding the lasing problem due to the coupling between the MDRs and the fluorescence emission, especially when pyrromethene 597-C8 is excited by the $532 \mathrm{~nm}$ line of a frequency-doubled Nd-Yag laser. Particular attention should be also paid to the significance of the temperature measured by LIF. In general, this temperature differs from the arithmetic volume averaged temperature, as the fluorescence signal is heavily weighted by the central region of the drop than by the region close to the surface. 
The measurement techniques developed in the frame of this study were able to quantify some of the effects due to the aerodynamic interactions between closely spaced droplets. Measurements of the size and temperature of the droplets showed a clear decrease in the rate of heating and evaporation compared to the isolated droplet. The dimensionless distance between the drops is an important factor to describe the effects of droplet interactions but is not the only one to be considered. The volatility of the liquid fuel also has a strong influence. Based on these first results, it may be assumed that the Stefan flow protects the droplets that are vaporizing at a fast rate from the disturbances of the external flow induced by neighbor droplets.

\section{References}

Abramzon B, Sirignano WA (1989) Droplet vaporization model for spray combustion calculations. Int $\mathrm{J}$ Heat Mass Transf 32:1605-1618

Castanet G, Lebouche M, Lemoine F (2005) Heat and mass transfer of combusting monodisperse droplets in a linear stream. Int $\mathbf{J}$ Heat Mass Transf 48:3261-3275
Castanet G, Frackowiak B, Tropea C, Lemoine F (2011) Heat convection within evaporating droplets in strong aerodynamic interactions. Int J Heat Mass Transf 54:3267-3276

Deprédurand V, Miron P, Labergue A, Wolff M, Castanet G, Lemoine F (2008) A temperature-sensitive tracer suitable for two-colour laser-induced fluorescence thermometry applied to evaporating fuel droplets. Meas Sci Technol 19:105403

Deprédurand V, Castanet G, Lemoine F (2010) Heat and mass transfer in evaporating droplets in interaction: influence of the fuel. Int $\mathrm{J}$ Heat Mass Transf 53:3495-3502

Frohn A, Roth N (2000) Section 6.4.4 microexplosions. In: Dynamics of droplets. Springer, Heidelberg, pp 229

Lavieille P, Lemoine F, Lavergne G, Lebouché M (2001) Evaporating and combusting droplet temperature measurements using twocolor laser-induced fluorescence. Exp Fluids 31:45-55

Lavieille P, Delconte A, Blondel D, Lebouché M, Lemoine F (2004) Non-intrusive temperature measurements using three-color laserinduced fluorescence. Exp Fluids 36:706-716

Lemoine F, Castanet G (2013) Temperature and chemical composition of droplets by optical measurement techniques: a state-ofthe-art review. Exp Fluids 54:1-34

Maqua C, Castanet G, Lemoine F, Doué N, Lavergne G (2006) Temperature measurements of binary droplets using three-color laserinduced fluorescence. Exp Fluids 40:786-797

Virepinte JF, Biscos Y, Lavergne G, Magre P, Collin G (2000) A rectilinear droplet stream in combustion: droplet and gas phase properties. Combust Sci Technol 150:143-159 